\title{
Synthesis and Molecular Docking Studies of Novel 2-(2-Amino-6-Phenyl-4-Pyrimidinylamino) Ethanol Derivatives: Using Ring-Opening Reactions of Cyclic Ketene- $\mathbf{N}, \mathbf{O}$-Acetal
}

\author{
G.M.V.N.A.R. RAVI KUMAR and ARUMUGAM THANGAMANI* \\ Department of Chemistry, Karpagam University, Karpagam academy of higher education, \\ Coimbatore-641021, Tamil Nadu, India. \\ *Corresponding author E-mail: Thangabell2003@gmail.com \\ http://dx.doi.org/10.13005/ojc/330361
}

(Received: May 03, 2017; Accepted: May 31, 2017)

\begin{abstract}
A series of six novel 2-(2-amino-6-phenyl-4-pyrimidinylamino) ethanol derivatives have been synthesized starting from commercially available substituted acetophenones via Oxoketene Dithioacetals with high yields. Ketene dithioacetal store act with 2-aminoethanol or l-amino-2-propanol to afford the corresponding substituted 2-methyleneoxazolidines which are utilized in the synthesis of 6-aryl-2-amino-4-pyrimidinamine Derivatives. The molecular docking studies revealed that all the synthesized compounds best fit into the active site of HDAC2, anti-cancer protein.
\end{abstract}

Keywords: 2-amino-pyrimidine Derivatives, Ketene dithioacetals, Cyclic Ketene N, O-acetal, molecular docking

\section{INTRODUCTION}

Cancer is a primary cause of death globally, impacting above 14 million people per annum. Among various types of cancers, Colon cancer is the most common type. Throughout the world, this cancer is the most frequently diagnosed (second in women and third in men) and it consistently lies within the five most serious types of cancer each year. This fact clearly emphasizes the need for detection of novel chemotherapeutic agents with potent anticancer activities. The core moiety of several anticancer agents comprised the pyrimidine nucleus which acts as tyrosine kinase inhibitors. Pyrimidine containing compounds are renowned for their biological activities due to the existence of pyrimidine base in genetic material of cells particularly in thymine, cytosine and uracil, which form the building blocks of DNA and RNA. 6-aryl2,4-Diamino pyrimidine (I) derivatives are known to exhibit important biological activities such as antifilarial to poisomerase II inhibitors ${ }^{1} \mathrm{MTH}-1$ inhibitors for treating cancer ${ }^{3}$ anti-nflammators ${ }^{4}$ and they are known to show potential anti plasmodial agents $^{2}$. But the synthesis and biological activity of 2-(2-amino-6-phenyl-4-pyrimidinylamino)ethanol 


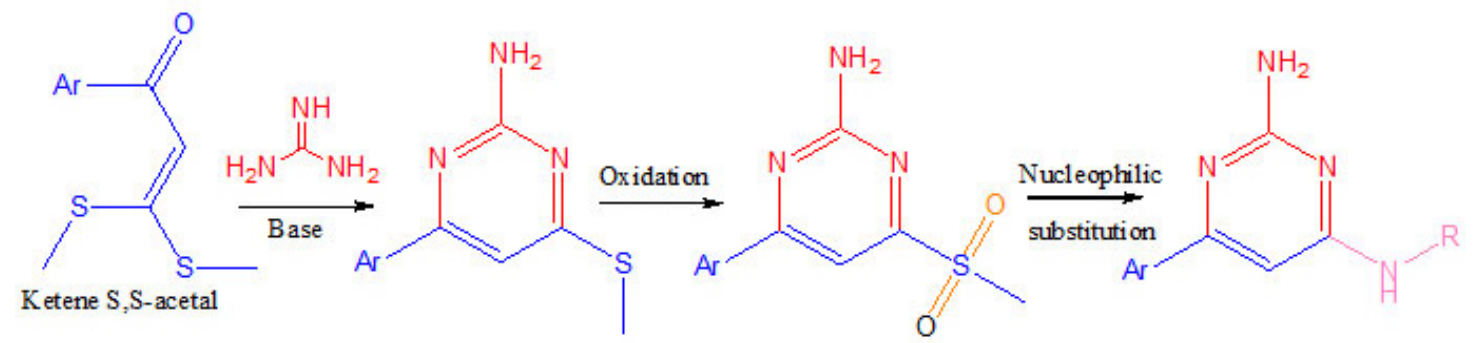

Scheme 1: Earlier methodology

(I) derivatives are not much investigated. These pyrimidine derivatives were previously synthesized from ketene S, Sacetals by treating with guanidine ${ }^{11}$. And these pyrimidine derivatives having sulfide group was oxidized to to sulphone and substituted with amine $^{1,2,6}$ (scheme-1). But the main disadvantage involves nucleophilic Substitution on pyrmidine ring having sulphone group need conditions.

The other drawback is more number of steps are required in this strategy, thereby combined yield seems to be less (scheme 1).

\section{AIM AND OBJECTIVES}

The 2-(2-amino-6-phenyl-4-pyrimidinyl amino) ethanol derivatives were planned to synthesize in the aim of exploring their anticancer, antibacterial and antimicrobial activity. Here by, we are proposing a versatile novel method to synthesize these compounds in very high yields (via $\alpha$-Oxoketene Dithioacetals) from commercially available and cheap starting materials such as acetophenones. Here, we describe the synthesis of some new heterocyclic ketene $\mathrm{N}, \mathrm{O}$-acetals and their reactions with guanidine hydrochloride.

\section{Retro synthetic analysis}

2-(2-amino-6-aryl-4-pyrimidinamino) ethanol (I) derivatives could be synthesized from guanidine and 2-Oxazolidin-2-ylidene-1phenylethanone (II), five-membered cyclic ketene$\mathrm{N}, \mathrm{O}-$ acetals. These five-membered cyclic ketene$\mathrm{N}, \mathrm{O}-$ acetals can be achieved from commercially available substituted acetophenones through keteneS,S-acetals (Scheme 2).

\section{RESULTS AND DISCUSSION}

The synthetic strategy started from the commercially available substituted acetophenones. The substituted acetophenones were treated with $\mathrm{NaH}$ and $\mathrm{CS}_{2}$ followed by methylation using Mel, resulted the $\alpha$-Oxoketene dithioacetals in a one pot reaction ${ }^{7-9}$ (Scheme 1). Further these ketene dithioacetal were treated with 2-ethanolamine under basic conditions yielded the required Cyclic $\mathrm{N}, \mathrm{O}-$ ketenecetals $^{10}(3 \mathrm{a}-3 \mathrm{f})$. From the yield values in the tabular result (table-1), it is clearly showing that ketene dithioacetals having electron donating groups on aromatic ring are much favorable for the reaction. Whereas ketene dithioacetals having electron withdrawing groups on aromatic ring has comparably less reactivity, because of the delocalization of the electron cloud and stabilsation of the molecule. ${ }^{1} \mathrm{H}$ and ${ }^{13} \mathrm{C}$-NMR spectra of the synthesized cyclic N,Oketenecetals (3a-3e) shows the presence of only one set of signals, representing that these compounds are not a mixture of $E$ and $Z$ isomers. Because of the presence of intra molecular hydrogen bond (as shown in figure 1)these compounds (3a-3e) are purely in the form of $\mathrm{E}$-isomer ${ }^{9}$. The compound 3 fwas existed as a mixture of $E, Z$ isomers in 10:1 ratio.

These Cyclic N,O-ketene acetals were made to react with guanidine hydrochloride using potassium tert. Butoxide as base resulted the required 2-(2-amino-6-aryl-4-pyrimidinamino) ethanol Derivatives (4a-4f). From the yield values in tabular result (table-2), it is showing that electron withdrawing groups on aromatic ring is favorable for the reaction. The structures of all the six compounds were fully characterized by ${ }^{1} \mathrm{H} N M R,{ }^{13} \mathrm{C}$ NMR, Mass and IR. 
<smiles>[R]c1cc([R3])c(C(C)=O)c([R])c1</smiles><smiles>CO[Mg]O[Mg]</smiles>

Substituted Acetophenone<smiles>[R]c1cc([R])c(C(=O)C=C(SC)SC)c([R])c1</smiles>

Ketene thioacetals<smiles>[R]c1cc([R2])c(C(=O)C=C2NCCO2)c([R2])c1</smiles>

Cyclic N,O-ketene ace 3a-3f

Table 1: Synthesis of Cyclic Ketone N2Oacetals

\begin{tabular}{lcccc}
\hline Compound & R1 & R2 & R3 & Yield \\
\hline 3a & H & OMe & OMe & $99 \%$ \\
3b & OMe & H & H & $98.6 \%$ \\
3c & H & H & H & $94.8 \%$ \\
3d & CL & H & H & $93.5 \%$ \\
3e & F & H & H & $93.1 \%$ \\
3f & NO2 & H & H & $60 \%$ \\
\hline
\end{tabular}<smiles>[R]c1cc([R])c(-c2cc(NCCO)nc(N)n2)c([R])c1</smiles>

2-(2-amino-6-arylpyrimidin -4-ylamino)ethanol<smiles>[R]c1cc([R])c(C(=O)C=C2NCCO2)c([R])c1</smiles>

II

cyclic

ketene-N,O-acetal<smiles>[R]c1cc([R])c(C(=O)C=C(SC)SC)c([R])c1</smiles>

Ketene S,S-acetals<smiles>[R]c1cc([R2])c(C(C)=O)c([R2])c1</smiles>

Substituted Acetophenone

\section{Scheme 2: Retrosynthesis Analysis}

\section{Plausible mechanism}

A postulated reaction pathway for the formation of product undergoes Michael addition with push-pull alkene, which was followed by intramolecular cyclization by the breakage of C-O bond to afford required products in good yields.

\section{Docking Studies \\ Materials and methods Ligand preparation}

Geometry optimized 2D structure of synthesized molecules was sketched and prepared for docking by using Ligprep, a versatile program of Schrödinger suite 2015 and minimized using OPLS-2005 force field. A total 10 conformation were generated.

\section{Protein preparation}

Crystal co-ordinates for HDAC2(PDB ID:3MAX) were taken from protein databank (http://www.rcsb.org). Protein was prepared for docking studies using protein prep wizard of Schrödinger 2016 (Maestro 11, Schrödinger, LLC, New York, NY). Bond orders and formal charges were added for hetero groups, and hydrogen's were added to all atoms in the system. Water molecules with in $5 \mathrm{~A}^{0}$ distance were removed. For each structure, a brief relaxation was performed using an all-atom constrained minimization carried out with the Impact Refinement module using the OPLS2005 force field to alleviate steric clashes that may exist in the original PDB structure. The minimization was terminated when the energy converged or the RMSD reached a maximum cut off of $0.30 \AA$.

Benzamide with Grid co-ordinates of X: 66.51, Y: 29.55, Z: 1.38 in anticancer HDAC2 protein.

\section{Glide Docking}

All the synthesized compounds (4a-4f) were docked into active site pockets of corresponding 


Tetene- $\mathrm{N}, \mathrm{O}$-acetal
Table 2: Synthesis of 2-(2-amino-6-aryl-4-
Compund

protein by using GLIDE ${ }^{13}$ module of Schrödinger. Standard precision docking was performed. A total of 10 ligand conformations were allowed and finally top score conformation was selected as active conformation. Molecules were analysed based on docking score, interacting amino acids, and hydrogen bonds (Table 3). $\mathrm{N}$-(2-aminophenyl) Benzamide was considered as standards for HDAC2 respectively.

\section{EXPERIMENTAL SECTION}

General procedure for the synthesis of Cyclic N,O-kenteneacetal (3a to $3 \mathrm{f}$ )

To the starting material (ketene diothioacetal) taken in DMF,2 equivalents of ethanolamine was added. Then 5 equivalents of sodium Hydride was added at 0 degrees and it was stirred at RT for 2 hours. Quenched the reaction mixture with Ice cold water and extracted with ethyl acetate to get the product. All the synthesized compounds were confirmed by ${ }^{1} \mathrm{H}$ and ${ }^{13} \mathrm{C}$-NMR spectral data.

General procedure for the synthesis of 2-(2amino-6-phenyl-4-pyrimidinylamino)ethanol (4a to $4 \mathrm{f}$ )

To the $500 \mathrm{mg}$ of Starting material (N,Oketene derivatives) taken in $10 \mathrm{ml}$ of DMSO and $10 \mathrm{ml}$ of toluene, two equivalents of guanidine. $\mathrm{HCl}$ and 2 equivalents potassium tert. But oxide was added at RT. it was stirred at $110^{\circ} \mathrm{C}$ for 5 hours. Quenched the reaction mixture with water and extracted with $10 \%$ methanol in chloroform to get the product, which was stirred with diethyl ether and filtered to get required product. All the synthesized compounds were confirmed by ${ }^{1} \mathrm{H}$ NMR, ${ }^{13} \mathrm{C}-\mathrm{NMR}$, Mass and IR.

2-(2-amino-6-(2,6-dimethoxyphenyl)pyrimidin-4ylamino)ethanol (4a)

M.P. $190^{\circ} \mathrm{C}$; $\mathrm{Rf}$ value 0.45 (in $10 \%$ Methanol in DCM); $\mathrm{H}^{1} \mathrm{NMR}\left(500 \mathrm{MHz}, \mathrm{DMSO}-\mathrm{d} 6,27^{\circ} \mathrm{C}\right)$

$\delta$ ppm 3.3(2H, bs), $3.5(2 \mathrm{H}, \mathrm{bs}), 3.64(6 \mathrm{H}, \mathrm{s}), 4.72$ $\left(1 \mathrm{H}, \mathrm{s}, \mathrm{D}_{2} \mathrm{O}\right.$ exchangeable), $5.59(1 \mathrm{H}, \mathrm{s}), 5.82(2 \mathrm{H}, \mathrm{s}$, $\mathrm{NH} 2, \mathrm{D}_{2} \mathrm{O}$ exchangeable), $6.65(2 \mathrm{H}, \mathrm{d}, \mathrm{J}=8.5), 6.74$ $\left(1 \mathrm{H}, \mathrm{bs}, \mathrm{D}_{2} \mathrm{O}\right.$ exchangeable $) 7.26(1 \mathrm{H}, \mathrm{t}, \mathrm{J}=8.5) ;{ }^{13} \mathrm{C}$ NMR $\left(400 \mathrm{MHz}, \mathrm{DMSO}-d_{6}, 2^{\circ} \mathrm{C}\right) \delta \mathrm{ppm} 42.5,55.4$ , 60.0 , 96.9, 103.9, 118.3, 129.0, 157.1, 159.7, 162.7, 163.1; ESI MS: [M++H] $291 \mathrm{~m} / \mathrm{z}$; IR: 3477 $\mathrm{cm}^{-1}, 3415 \mathrm{~cm}^{-1}, 3332 \mathrm{~cm}^{-1}, 3198 \mathrm{~cm}^{-1}, 2960 \mathrm{~cm}^{-1}$, $2929 \mathrm{~cm}^{-1}, 2853 \mathrm{~cm}^{-1}$

2-(2-amino-6-(4-methoxyphenyl)pyrimidin-4ylamino)ethanol (4b)

M.P. $148^{\circ} \mathrm{C}$; Rf value 0.50 (in $10 \%$ Mein DCM); ${ }^{1} \mathrm{H} \mathrm{NMR}(300 \mathrm{MHz}, \mathrm{DMSO})$ ä ppm $3.3(2 \mathrm{H}, \mathrm{t})$, 
<smiles></smiles>

Intramolecular Hydrogen bonding

Fig. 1

Table 3: Docking scores

\begin{tabular}{ll}
\hline Compound Code & HDAC2 docking score \\
\hline $4 \mathrm{a}$ & -7.477 \\
4b & -5.686 \\
$4 \mathrm{c}$ & -7.945 \\
4d & -7.774 \\
4e & -7.725 \\
$4 \mathrm{f}$ & -7.998 \\
Standard [N-(2-aminophynel) & -13.015 \\
Benzamide] & \\
\hline
\end{tabular}

$3.4(2 \mathrm{H}, \mathrm{t}, \mathrm{J}=5.4), 3.77(3 \mathrm{H}, \mathrm{s}), 4.71(1 \mathrm{H}, \mathrm{t}, \mathrm{J}=5.1$, D2O exchangeable), $5.90\left(2 \mathrm{H}, \mathrm{s}, \mathrm{D}_{2} \mathrm{O}\right.$ exchangeable), $6.18(1 \mathrm{H}, \mathrm{s}), 6.76\left(1 \mathrm{H}, \mathrm{S}, \mathrm{D}_{2} \mathrm{O}\right.$ exchangeable), 6.96 $(2 \mathrm{H}, \mathrm{d}, \mathrm{J}=8.7), 7.83(2 \mathrm{H}, \mathrm{d}, \mathrm{J}=5.4) ;{ }^{13} \mathrm{C}$ NMR $(400 \mathrm{MHz}$, DMSO- $\left.d_{6}, 2^{\circ} \mathrm{C}\right) \delta \mathrm{ppm} 42.7,55.1,60.1,89.9,113.6$, 127.5, 130.6, 160.2, 163.2 , 164.1; IR: $3443 \mathrm{~cm}^{-1}$, $3315 \mathrm{~cm}^{-1}, 3218 \mathrm{~cm}^{-1}, 2987 \mathrm{~cm}^{-1}, 2956 \mathrm{~cm}^{-1}, 2937$ $\mathrm{cm}^{-1}, 2862 \mathrm{~cm}^{-1}$

\section{2-(2-amino-6-phenylpyrimidin-4-ylamino)ethanol} (4c)

M.P. $118^{\circ} \mathrm{C}$; Rf value 0.65 (in $10 \%$ Methanol in DCM) $\mathrm{H}^{1} \mathrm{NMR}\left(500 \mathrm{MHz}\right.$, DMSO-d6, $\left.27^{\circ} \mathrm{C}\right) \delta$ ppm 3.3(2H, t), $3.5(2 \mathrm{H}, \mathrm{dd}, \mathrm{J}=6, \mathrm{~J}=11.5), 4.72(1 \mathrm{H}$, $\mathrm{t}, \mathrm{J}=5$, Exchangeable proton), $6.02\left(2 \mathrm{H}, \mathrm{s},-\mathrm{NH}_{2}\right.$, Exchangeable proton), $6.27(1 \mathrm{H}, \mathrm{s}), 6.95(1 \mathrm{H}, \mathrm{bs}$, Exchangeable proton), 7.49 (2H, d, J=8.5), 7.91
$(2 \mathrm{H}, \mathrm{d}, \mathrm{J}=7.5) ;{ }^{13} \mathrm{C}$ NMR $\left(400 \mathrm{MHz}\right.$, DMSO- $\left.d_{6}, 25^{\circ} \mathrm{C}\right)$ $\delta$ ppm 42.7, 60.0, 90.9, 126.1, 127.2, 128.2, 128.9, 129.2, 138.3, 161.4, 163.4, 164.1; ESI MS: [M $\left.{ }^{+}+\mathrm{H}\right]$ $231 \mathrm{~m} / \mathrm{z}$; IR: $3386 \mathrm{~cm}^{-1}, 3325 \mathrm{~cm}^{-1}, 3221 \mathrm{~cm}^{-1}, 3110$ $\mathrm{cm}^{-1}, 2992 \mathrm{~cm}^{-1}, 2938 \mathrm{~cm}^{-1}, 2879 \mathrm{~cm}^{-1}$

\section{2-(2-amino-6-(4-chlorophenyl)pyrimidin-4- ylamino)ethanol (4d)}

M.P. $178^{\circ} \mathrm{C}$; $\mathrm{Rf}$ value 0.49 (in $10 \%$ Methanol in DCM); $\mathrm{H}^{1} \mathrm{NMR}\left(500 \mathrm{MHz}\right.$, DMSO-d6, 27 $\left.{ }^{\circ} \mathrm{C}\right)$ $\delta \mathrm{ppm} 3.3(2 \mathrm{H}, \mathrm{t}, \mathrm{J}=5.4), \quad 3.50-3.53(2 \mathrm{H}, \mathrm{m}), 4.73$ $\left(1 \mathrm{H}, \mathrm{t}, \mathrm{J}=5\right.$, Exchangeable proton), $5.97\left(2 \mathrm{H}, \mathrm{s},-\mathrm{NH}_{2}\right.$, exchangeable proton), $6.26(1 \mathrm{H}, \mathrm{S}), 6.87(1 \mathrm{H}, \mathrm{bs}$, exchangeable proton), 7.39-7.45 $(3 \mathrm{H}, \mathrm{m}), 7.99(2 \mathrm{H}$, $\mathrm{d}, \mathrm{J}=6.5) ;{ }^{13} \mathrm{C}$ NMR $\left(400 \mathrm{MHz}\right.$, DMSO- $\left.d_{6}, 25^{\circ} \mathrm{C}\right)$ $\delta$ ppm 42.7, 59.9, 67.4, 72.5 , 90.95, 127.9, 128.1, 128.3, 133.9, 137.1, 160.1, 163.3, 164.1. ESI MS: $\left[\mathrm{M}^{+}+\mathrm{H}\right] 265 \mathrm{~m} / \mathrm{z} ; \mathrm{IR}: 3403 \mathrm{~cm}^{-1}, 3317 \mathrm{~cm}^{-1}, 3217 \mathrm{~cm}^{-1}$, $3104 \mathrm{~cm}^{-1}, 2951 \mathrm{~cm}^{-1}, 2924 \mathrm{~cm}^{-1}, 2868 \mathrm{~cm}^{-1}$

\section{2-(2-amino-6-(4-fluorophenyl)pyrimidin-4- ylamino)ethanol (4e)}

M.P. $158-159^{\circ} \mathrm{C}$, Rf value 0.47 (in $10 \%$ Methanol in DCM) $\mathrm{H}^{1} \mathrm{NMR}(500 \mathrm{MHz}$, DMSO-d6, $\left.27^{\circ} \mathrm{C}\right) \delta \mathrm{ppm} 3.3(2 \mathrm{H}, \mathrm{t}, \mathrm{J}=5.4), 3.51(2 \mathrm{H}, \mathrm{t}, \mathrm{J}=5.9)$, $5.99\left(2 \mathrm{H}, \mathrm{S},-\mathrm{NH}_{2}\right.$, Exchangeable proton), 6.25 $(1 \mathrm{H}, \mathrm{s}), 6.90(1 \mathrm{H}, \mathrm{bs}$, Exchangeable proton), $7.26(2 \mathrm{H}$, $\mathrm{t}, \mathrm{J}=8.8), 7.94(2 \mathrm{H}, \mathrm{s}) ;{ }^{13} \mathrm{C}$ NMR $\left(400 \mathrm{MHz}\right.$, DMSO- $d_{6}$, $\left.25^{\circ} \mathrm{C}\right) \delta \mathrm{ppm} 42.14,60.0,91.0,115.2,128.4,134.8$ , 158.3, 160.3, 161.6, 163.3, 164.1, 164.2; ESI MS: $\left[\mathrm{M}^{+}+\mathrm{H}\right] 249 \mathrm{~m} / \mathrm{z} ; \mathrm{IR}: 3398 \mathrm{~cm}^{-1}, 3221 \mathrm{~cm}^{-1}, 3152 \mathrm{~cm}^{-1}$, $2994 \mathrm{~cm}^{-1}, 2953 \mathrm{~cm}^{-1}, 2937 \mathrm{~cm}^{-1}, 2879 \mathrm{~cm}^{-1}$

\section{2-(2-amino-6-(4-nitrophenyl)pyrimidin-4-ylamino) ethanol (4f)}

M.P. $192^{\circ} \mathrm{C}$, Rf value 0.38 (in $10 \%$ Methanol in DCM); $\mathrm{H}^{1} \mathrm{NMR}\left(300 \mathrm{MHz}, \mathrm{DMSO}-\mathrm{d} 6,27^{\circ} \mathrm{C}\right)$ $\delta$ ppm $3.5(2 \mathrm{H}, \mathrm{t}), 3.79(2 \mathrm{H}, \mathrm{t}, \mathrm{J}=3), 4.91(2 \mathrm{H}, \mathrm{s}), 5.83$ $(1 \mathrm{H}, \mathrm{bs}), 6.28(1 \mathrm{H}, \mathrm{S}), 7.97(1 \mathrm{H}, \mathrm{d}), 8.08(2 \mathrm{H}, \mathrm{d}, \mathrm{J}=4.5)$, $8.26(2 \mathrm{H}, \mathrm{d}, \mathrm{J}=4.8) ;{ }^{13} \mathrm{C}$ NMR $\left(400 \mathrm{MHz}\right.$, DMSO- $d_{6}$,

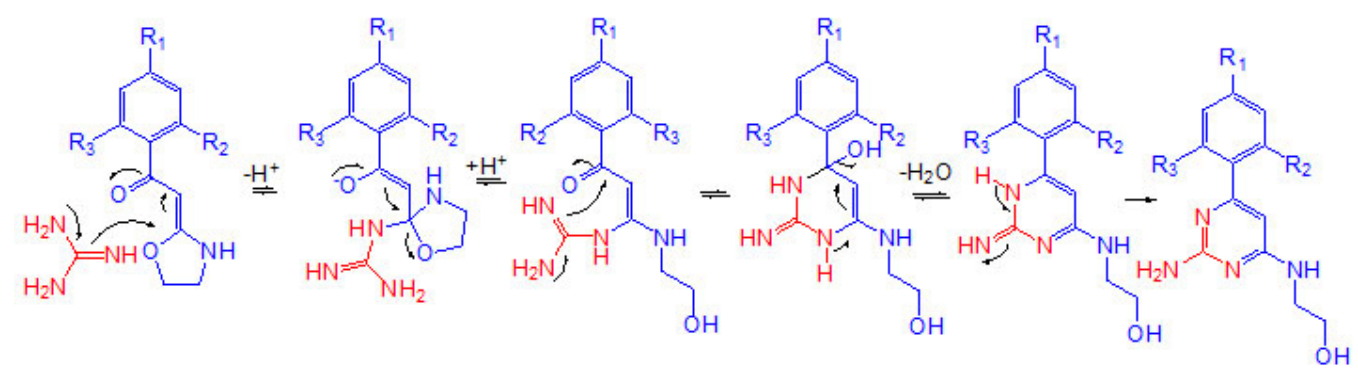

Fig. 2: Plausible mechanism 


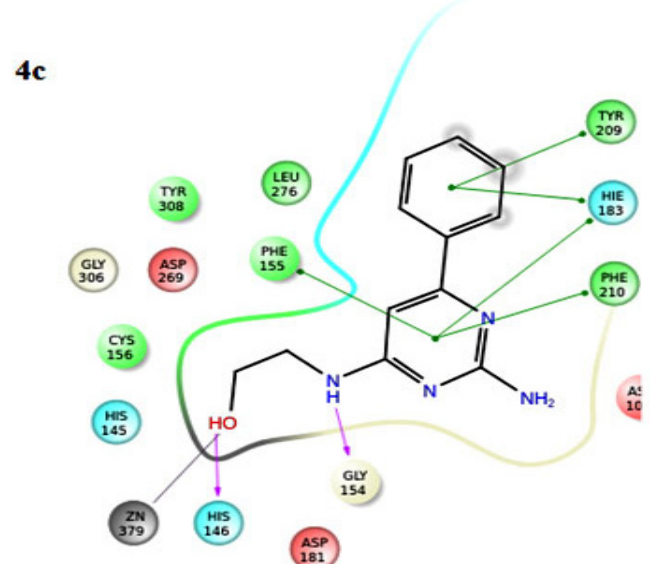

4d<smiles></smiles>

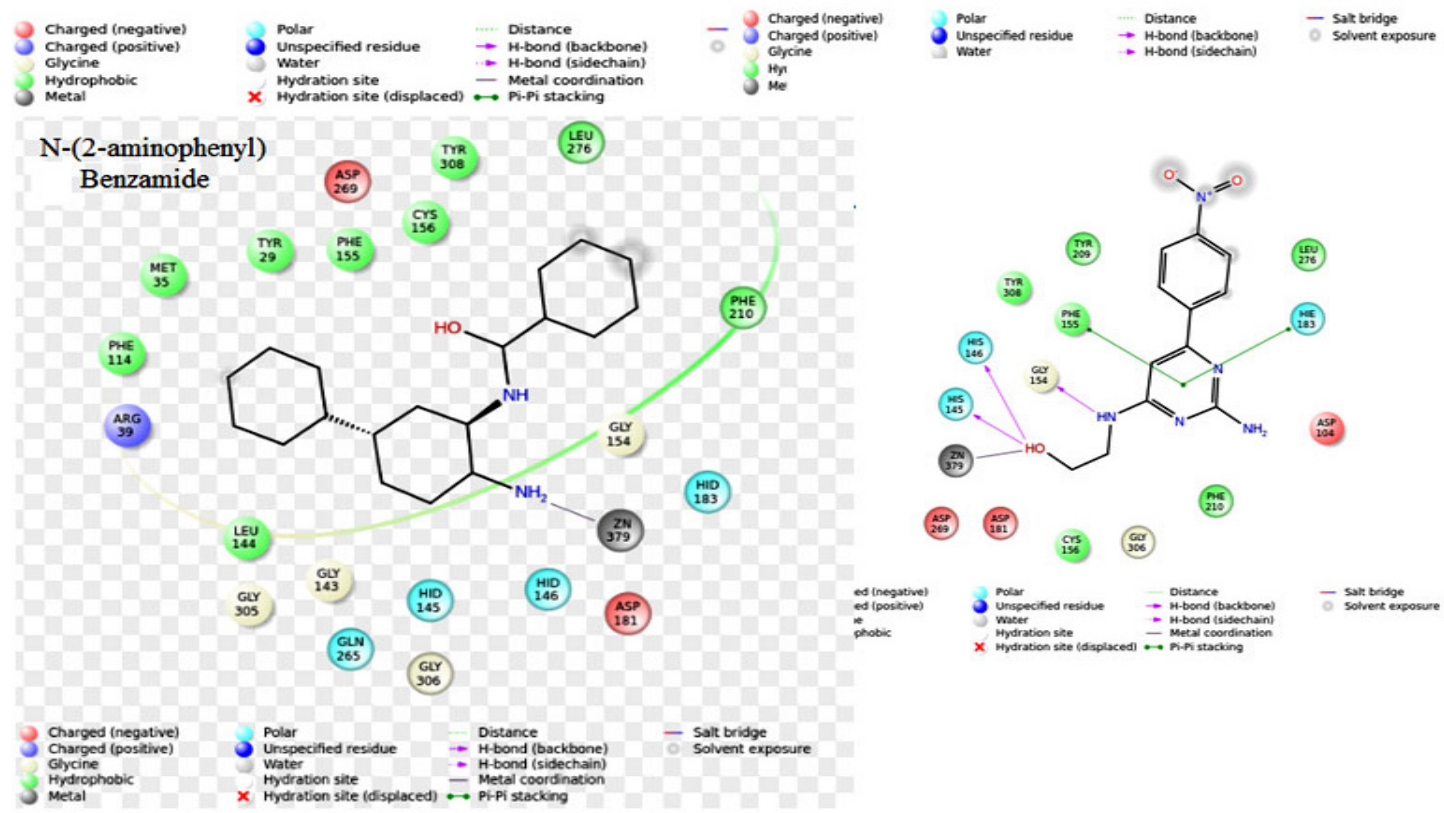

Fig. 3: Ligand diagrams

$\left.2^{\circ} \mathrm{C}\right) \delta \mathrm{ppm} 42.1,59.8,94.9,123.6,123.3,144.6$, 147.8, 163.5, 163.8, 164.2; ESI MS: [M++H] 275 m/z; IR: $3441 \mathrm{~cm}^{-1}, 2954 \mathrm{~cm}^{-1}, 2918 \mathrm{~cm}^{-1}$

(E)-1-(4-methoxyphenyl)-2-(oxazolidin-2-ylidene) ethanone (Compound 3b)

$\mathrm{H}^{1} \mathrm{NMR}\left(300 \mathrm{MHz}\right.$, DMSO-d6, 27 $\left.{ }^{\circ} \mathrm{C}\right) \delta \mathrm{ppm}$ $3.70(2 \mathrm{H}, \mathrm{t}, \mathrm{J}=8.4), 3.78(3 \mathrm{H}, \mathrm{s}), 4.45(2 \mathrm{H}, \mathrm{t}, \mathrm{J}=8.4)$, $5.50(1 \mathrm{H}, \mathrm{s}), 6.93(2 \mathrm{H}, \mathrm{d}, \mathrm{J}=8.7), 7.79(2 \mathrm{H}, \mathrm{d}, \mathrm{J}=8.7)$, $9.72(1 \mathrm{H}, \mathrm{bs}) ; \mathrm{C}^{13} \mathrm{NMR}\left(400 \mathrm{MHz}, \mathrm{DMSO}-\mathrm{d} 6,25^{\circ} \mathrm{C}\right)$ $\delta$ ppm 43.015, 55.501, 67.0, 67.2, 72.0, 113.2, 128.2, 128.2, 132.5, 161.0, 169.3, 184.2.
(E)-2-(oxazolidin-2-ylidene)-1-phenylethanone (compound 3c)

$\mathrm{H}^{1} \mathrm{NMR}\left(400 \mathrm{MHz}, \mathrm{DMSO}-\mathrm{d} 6,25^{\circ} \mathrm{C}\right) \delta \mathrm{ppm}$ $3.72(2 \mathrm{H}, \mathrm{t}, \mathrm{J}=8.4), 4.46(2 \mathrm{H}, \mathrm{t}, \mathrm{J}=8.4), 5.55(1 \mathrm{H}, \mathrm{s})$, $7.41(3 \mathrm{H}, \mathrm{m}), 7.83(2 \mathrm{H}, \mathrm{d}, \mathrm{J}=6.4), 9.81(1 \mathrm{H}, \mathrm{bs}) ; \mathrm{C}^{13}$ NMR (400MHz, DMSO-d6, $\left.25^{\circ} \mathrm{C}\right) \delta$ ppm 46.8, 67.3, 72.5, 126.4, 128.3, 133.2, 139.9, 169.6, 184.7.

(E)-1-(4-chlorophenyl)-2-(oxazolidin-2-ylidene) ethanone (compound $3 d$ )

$\mathrm{H}^{1} \mathrm{NMR}\left(400 \mathrm{MHz}, \mathrm{DMSO}-\mathrm{d} 6,25^{\circ} \mathrm{C}\right) \delta \mathrm{ppm}$ $3.72(2 \mathrm{H}, \mathrm{t}, \mathrm{J}=8.4), 4.47(2 \mathrm{H}, \mathrm{t}, \mathrm{J}=8.4), 5.53(1 \mathrm{H}, \mathrm{s})$, $7.42(2 \mathrm{H}, \mathrm{d}, \mathrm{J}=8.4), 7.83$ (2H, dd, J=8.8, J=2.0), 9.81 
$(1 \mathrm{H}, \mathrm{bs}) ; \mathrm{C}^{13} \mathrm{NMR}\left(400 \mathrm{MHz}, \mathrm{DMSO}-\mathrm{d} 6,25^{\circ} \mathrm{C}\right) \delta \mathrm{ppm}$ 43.0, 67.4, 72.5, 128.0, 128.3, 135.0, 138.6, 169.6, 183.2 .

\section{(E)-1-(4-fluorophenyl)-2-(oxazolidin-2-ylidene)} ethanone (compound $3 e$ )

$\mathrm{H}^{1} \mathrm{NMR}\left(400 \mathrm{MHz}, \mathrm{DMSO}-\mathrm{d} 6,25^{\circ} \mathrm{C}\right) \delta$ ppm $3.72(2 \mathrm{H}, \mathrm{t}, \mathrm{J}=8.4), 4.469(2 \mathrm{H}, \mathrm{t}, \mathrm{J}=8.4), 5.53$ $(1 \mathrm{H}, \mathrm{s}), 7.19(2 \mathrm{H}, \mathrm{d}, \mathrm{t}=8.8), 7.87(2 \mathrm{H}, \mathrm{m}), 9.76(1 \mathrm{H}$, bs); $\mathrm{C}^{13} \mathrm{NMR}\left(400 \mathrm{MHz}\right.$, DMSO-d6, $\left.25^{\circ} \mathrm{C}\right) \delta \mathrm{ppm}$ 43.054, 67.3, 72.3, 114.7, 114.9, 128.8, 128.9, 136.4, 136.4, 162.2, 164.6, 169.6, 183.4 .

(E,Z)-1-(4-nitrophenyl)-2-(oxazolidin-2-ylidene) ethanone (compound $3 f$ as a mixture of $E, Z$ isomers in 10:1 ratio)

$\mathrm{H}^{1} \mathrm{NMR}\left(400 \mathrm{MHz}, \mathrm{DMSO}-\mathrm{d} 6,25^{\circ} \mathrm{C}\right) \delta$ ppm $3.71(0.2 \mathrm{H}, \mathrm{t}, \mathrm{J}=8), 3.75(2 \mathrm{H}, \mathrm{t}, \mathrm{J}=8.8), 4.45$ $(0.2 \mathrm{H}, \mathrm{t}, \mathrm{J}=8.4), 4.51(2 \mathrm{H}, \mathrm{t}, \mathrm{J}=8.8), 5.51(0.1 \mathrm{H}$, s), $5.61(1 \mathrm{H}, \mathrm{s}), 7.25(0.2 \mathrm{H}, \mathrm{dd}, \mathrm{J}=2, \mathrm{~J}=6.8), 7.75$ $(0.2 \mathrm{H}, \mathrm{dd}, \mathrm{J}=2, \mathrm{~J}=6.8), 8.03-8.06(2 \mathrm{H}, \mathrm{m}), 8.21-8.24$ $(2 \mathrm{H}, \mathrm{m}), 9.76(0.1 \mathrm{H}, \mathrm{bs}), 9.92(1 \mathrm{H}, \mathrm{bs}) ; \mathrm{C}^{13} \mathrm{NMR}$
(400MHz, DMSO-d6, $\left.25^{\circ} \mathrm{C}\right) \delta$ ppm 43.0, 43.1, 67.6, 73.6, 123.3, 124.8, 127, 127.7, 145.4, 148.3, 169.8, 182.0 .

\section{CONCLUSION}

Six novel pyrimidine derivatives ( $4 a$ to 4f) were prepared and characterized by IR, Mass, ${ }^{1} \mathrm{H}$ and ${ }^{13} \mathrm{C}-\mathrm{NMR}$ spectral data. In conclusion, we have successfully synthesized 2-(2-amino-6-aryl-4pyrimidinamino) ethanol Derivatives. The molecular docking studies for the synthesized compounds revealed that all the synthesized compounds best fit into the active site of HDAC2. Compound 4c, $4 d$, $4 f$ is showing better results.

\section{ACKNOWLEDGEMENTS}

The authors expressed their gratitude to Dr. M. Mallika, Department of Medicinal Chemistry, NIPER- Hyderabad, for providing docking facility for the present research work.

\section{REFERENCES}

1. Katiyar, Sanjay Babu et al; Bioorganic \& Medicinal Chemistry Letters 2005, 15(1), 17.5n.

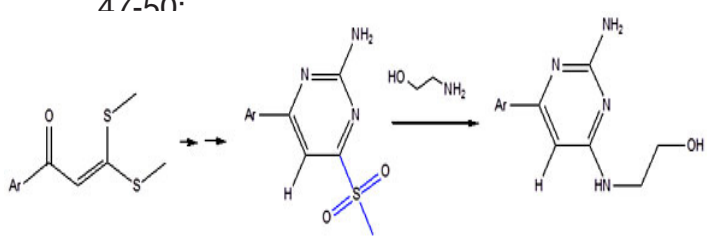

2. RajaniGiridhar, Riyaj S. Tamboli, Dhaval G. Prajapati, SanketSoni, Sarita Gupta, M. R. Yadav; Med Chem Res 2013, 22, 33093315

3. Scobie, Martin; Wallner, Olov; Koolmeister, Tobias; Vallin, Karl Sven Axel; Henriksson, Carl Martin; Jacques, Sylvain; Homan, Evert; Helleday, Thomas; PCT Int. Appl.WO 2015187088 A1, 2015

4. Love, Christopher John et al; PCT Int. Appl.2003015776, 27 Feb 2003

5. Cao, Sheldon Xiaodong et al; PCT Int. Appl.2002096867, 05 Dec 2002

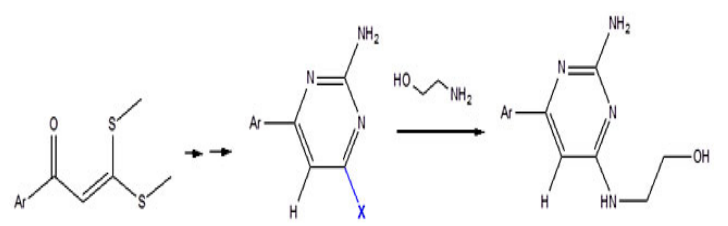

6. Daniela Cortese, Konstantin Chegaev, Stefano Guglielmo, Lan Z. Wang, Bernard T. Golding, Cline Cano and Roberta Fruttero; Chem.Med. Chem 2016, 11, 1705-1708

7. Potts, K. T.; Cipullo, M. J.; Ralli, P.; Theodoridis, G.; J. Org. Chem. 1982, 47(9), 3027;

8. Junjappa, H.; lla, H.; Asokan, C.V.; Tetrahedron 1990, 10, 5423;

9. Rudorf, W. D.; Schierhorn, A.; Augustin, M.; Tetrahedron 1979, 35, 551.

10. Yingquan Song, Hondamuni I. De Silva, William P. Henry, Guozhong Ye, Sabornie Chatterjee, Charles U. Pittman Jr.; Tetrahedron Letters 2011,52, 4507-4511

11. Singh, O. M.; Ila, H.; Junjappa, H.; Indian Journal of Chemistry - Section B: Organic 
and Medicinal Chemistry, 1997, 36(12), 11231125.

12. National Committee for Clinical Laboratory Standards 1993a. Performance Standards for Antimicrobial Disk Susceptibility Tests-Fifth Edition: Approved Standard M2-A5. NCCLS,
Villanova, PA.

13. Friesner, R. A.; Murphy, R. B.; Repasky, M. P.; Frye, L. L.; Greenwood, J. R.; Halgren,T. A.; Sanschagrin, P. C.; Mainz, D. T.; J. Med. Chem. 2006, 49, 6177-6196 